\title{
EFECTOS DE LA GESTIÓN DEL CONOCIMIENTO EN EL RENDIMIENTO DE LAS PYMES DE LA CIUDAD DE PEREIRA, DURANTE 2019
}

Vicki Lorena Bartolo Ladino*, Claudia Catalina Saldarriaga Aguirre**

\section{Resumen}

Las pequeñas y medianas empresas (pymes) se ven enfrentadas a un entorno cada vez más exigente, en donde el conocimiento se vuelve un recurso que permite desarrollar estrategias y capacidades con mejores beneficios a estas organizaciones; por tanto, una adecuada gestión del conocimiento (GC) que es el eje central de la investigación, donde se revisaron los efectos que tiene la gestión del conocimiento en el rendimiento en las pymes, que contribuirá a la mejora de la competitividad empresarial. Se realizó una investigación concluyente, descriptiva con un enfoque cualitativo y cuantitativo a una muestra de 352 pymes de la ciudad de Pereira. Los resultados permiten comprobar el efecto positivo que ejerce la GC sobre el rendimiento en las pymes de la ciudad de Pereira.

* Estudiante, Administración de Empresas, Fundación Universitaria del Área Andina. Correo: vbartolo@estudiantes.areandina.edu.co

* Estudiante, Administración de Empresas, Fundación Universitaria del Área Andina. Correo: csaldarriaga3@estudiantes.areandina.edu.co 


\section{Introducción}

Las pymes desempeñan un papel muy importante en la economía de un país. El informe presentado por la Comisión Europea (2015), expone que las microempresas y las pymes son el motor de la economía no solo europea sino también mundial. Impulsan la generación de nuevos puestos de trabajo, el crecimiento y fortalecimiento económico de los países, además de asegurar la estabilidad social. Según afirman Lahaba y Santos (2001) en su artículo, donde resaltan que para las compañías, la gestión del conocimiento (GC) permite el aumento del conocimiento, con el fin de ganar competitividad en los mercados. Compartir conocimiento en la empresa, permite fortalecer el know-how, aportando un valor a la misma, representado en conocimiento e incremento de los indicadores de rentabilidad y eficacia.

La GC tiene sus inicios en diferentes campos, las organizaciones deben usar procedimientos que les ayude a contribuir a la gestión para la permanencia de esta en el mercado; es así como esta investigación se centra en el análisis del efecto que ejerce la GC sobre el rendimiento, generando con ello una importante contribución teórica y empírica para que las empresas puedan tomar acciones que contribuyan al desarrollo del capital humano y a su competitividad.

Para cumplir con el objetivo de investigación, se realizó un estudio empírico a 352 pymes establecidas en la ciudad de Pereira, Risaralda. El estudio se desarrolló mediante la técnica estadística de regresión lineal por MCO (mínimos cuadrados ordinarios).

\section{Materiales y métodos}

El tipo de investigación es concluyente descriptiva con un enfoque de metodología cualitativa y cuantitativa, teniendo como centro de información la base de datos proporcionada por el DANE del 2017, a una muestra de 352 pymes de la ciudad de Pereira de los sectores servicios, industria y comercio que contaran con hasta 200 empleados, y activos totales mayores a 501 salarios mínimos legales vigentes (smlv) y hasta $30000 \mathrm{smlv}$. Se recogió la información por medio de encuestas, con un cuestionario autoadministrado dirigido al gerente de la empresa entre mayo y junio de 2019.

El cuestionario aplicado se basó en el estudio de Enrique (2017) y adaptado a nuestro objeto de estudio.

\section{Medición de variables}

Se midieron las siguientes variables: antigüedad de la empresa (años de funcionamiento), tamaño (número de trabajadores), GC y rendimiento. La información obtenida de la GC permitió medir variables como la adquisición del conocimiento externo (ACE), adquisición del conocimiento interno (ACI), transferencia del conocimiento (TC), explotación del conocimiento (EC), cultura organizacional (CO).

El rendimiento se midió a partir de la propuesta de Quinn (1983), con el empleo de indicadores a partir de la percepción del gerente de su posición competitiva sobre el rendimiento en los modelos de las relaciones humanas (MRH), modelo de sistema abierto (MSA), modelo 
racional (MR) y modelo de procesos internos (MPI). Las variables medidas se indican en el anexo A, correspondiente a la encuesta.

\section{Modelo estructural}

Para validar las hipótesis formuladas en la investigación y verificar la relación entre el rendimiento y la GC, se utilizó el modelo de regresión lineal por MCO. Para hacer el contraste de las hipótesis de la investigación se desarrollaron los modelos de regresión lineal a través del diseño de ecuaciones, tales como:

\section{Rendimiento $=b_{0}+b_{1}$ Tamaño $_{i}+b_{2} E-$ $\operatorname{dad}_{\mathrm{i}}+\mathrm{b}_{3} \mathrm{GC}+\mathfrak{E}_{\mathrm{i}}$ \\ Dependientes \\ Rto $_{i}=$ MPI, MSA, MR, MRH, rendi- miento global.}

Tamaño Número de empleados

Edad Añosdefuncionamiento

\section{Explicativa}

GC, ACE, ACI, TC, EC, CO, gestión del conocimiento global.

\section{Resultados}

\section{Validación de hipótesis}

Para estudiar el efecto que ejerce la GC sobre el rendimiento de la pyme, se analizó el coeficiente $b_{3}$ de la variable GC y su valor t-student asociado para saber si esta influencia es significativa (véase tabla 1).

La tabla 1 muestra las relaciones entre la GC global y el rendimiento. Se comprobó que existe una relación significativa positiva en todas las variables de GC con el rendimiento. Se verifican las hipótesis planteadas que indican que a mayor uso de la GC, mayor es el rendimiento que obtiene la pyme de la ciudad de Pereira; explicado por medio del coeficiente de $b_{3}(0,504)$ positivo y significativo. Las variables ACE, ACI, TC, EC y CO, también indican por separado que un mayor uso permite un mayor rendimiento en la pyme de la ciudad de Pereira y en mayor medida la EC con $b_{3}(0,456)$. Se pudo comprobar que las variables control edad y tamaño no ejercen influencia sobre el rendimiento de la pyme en Pereira.

Tabla 1. Relación entre las variables (GC y rendimiento)

\begin{tabular}{lcccc}
\hline \multicolumn{1}{c}{ Variables } & Valor & Valor $\mathbf{t}$ & Valor $f$ & $\mathbf{R}^{\mathbf{2}}$ Ajustado \\
\hline GC--> Rendimiento & 0,504 & 10,919 & 39,908 & 0,252 \\
\hline ACE--> Rendimiento & 0,288 & 6,147 & 12,738 & 0,092 \\
\hline ACl--> Rendimiento & 0,377 & 9,355 & 29,236 & 0,195 \\
\hline TC--> Rendimiento & 0,349 & 8,999 & 27,055 & 0,183 \\
\hline EC--> Rendimiento & 0,456 & 10,706 & 38,27 & 0,243 \\
\hline CO--> Rendimiento & 0,291 & 8,725 & 25,434 & 0,174 \\
\hline
\end{tabular}


Tabla 2. Relación entre GC y modelos de rendimiento (modelo proceso interno, modelo sistema abierto, modelo racional, modelo relaciones humanas)

\begin{tabular}{lcccc}
\hline \multicolumn{1}{c}{ Variables } & Valor $\mathbf{~}$ & Valor $\mathbf{t}$ & Valor $f$ & $\mathbf{R}^{\mathbf{2}}$ Ajustado \\
\hline GC-->MPI & 0,555 & 10,347 & 36,193 & 0,233 \\
\hline ACE--> MSA & 0,476 & 8,894 & 27,720 & 0,188 \\
\hline ACl--> MR & 0,451 & 6,878 & 17,058 & 0,122 \\
\hline TC-> MRH & 0,535 & 7,357 & 19,293 & 0,137 \\
\hline
\end{tabular}

La tabla 2 muestra la relación entre la GC y los modelos de rendimiento proceso interno, sistema abierto, racional y relaciones humanas, donde se hicieron todas las pruebas estadísticas y se obtiene un efecto positivo y significativo; es decir, que se concluyó que a mayor grado de utilización de GC, mayor es el rendimiento en todos los modelos de rendimiento de las pymes en la ciudad de Pereira, y mayor influencia MPI con coeficiente $b_{3}(0,555)$. Se confirma una vez más las hipótesis planteadas. Entre tanto, la variable control edad y tamaño no ejerce influencia sobre el rendimiento en los modelos de rendimiento.

\section{Discusión}

De acuerdo con los resultados se afirma que la GC es de gran relevancia para las pymes: contribuye a mejorar el rendimiento, permite el equilibrio de los objetivos y los medios empleados para lograrlos; mejora las competencias, la eficiencia y la toma de decisiones adecuada, el aprendizaje y la innovación, factores críticos para operar en condiciones exigentes dentro y fuera de la organización (Arceo y Salvador, 2006). Las organizaciones alcanzan valor a partir del conocimiento $y$, a su vez, generan

92 ventajas competitivas sostenibles y mayores rendimientos.

\section{Agradecimientos}

Agradecemos, en primer lugar, a Dios, por permitirnos vivir esta grata experiencia, por brindarnos sabiduría, paciencia y darnos la fuerza para no rendirnos y culminar de la mejor manera esta investigación.

A las profesoras Neise Vanegas Nieto y Luz Adriana Henao Mejía, expresar nuestros más profundos agradecimientos, por su confianza ofrecida hacia nosotras, por su paciencia, tiempo, conocimientos, y dedicación en todo momento y por hacer posible la realización de esta importante investigación.

A nuestra bella familia por la paciencia, amor y ánimo ofrecidos cada día en este proyecto, por brindarnos la confianza, motivación y acompañamiento en cada paso que damos en nuestra vida.

Asimismo, agradecemos a nuestras compañeras de carrera: Diana Moncada y Natalia Castaño, por compartir con nosotras sus experiencias y por apoyarnos con sus aportes.

Y, por último, a la Fundación Universitaria del Área Andina, por permitirnos formar parte de su familia, por orientarnos $\mathrm{y}$ brindarnos las herramientas y conoci- 
mientos necesarios para ser cada día mejores personas y excelentes profesionales.

\section{Referencias}

1. Arceo Moheno, G., \& Salvador Vallès, R. (septiembre de 2006). La gestión del conocimiento, la innovación y las tecnologías y comunicaciones en las pequeñas y medianas empresas. https://cutt.ly/zuEzz7p

2. Bedoya, M. P. (15 de julio de 2019). Eje Cafetero, crecimiento empresarial. Especiales La República. http://cort.as/-Suh6

3. Cadavid, J. I. (2019). Crecimiento empresarial. Eje Cafetero. La República.

4. Chiavenato. (2004). Introducción a la teoría general de la administración. México.

5. Comisión Europea. (2015). Guía del usuario sobre la definición del concepto de pyme. Luxemburgo. http://cort.as/-EhFr

6. Davenport, T., \& Prusak, L. (2001). Conocimiento en acción. Cómo las organizaciones manejan lo que saben. Buenos Aires.

7. Enrique, V. J. (2017). La gestión del conocimiento y las TIC, su efecto en la innovación y rendimiento de la pyme: un estudio empírico. https://cutt.ly/1uEkwpl

8. Figueroa, R. V. (2013). Conocimiento táctico e implícito.

9. Garzón, R. (2018). Programa sobre operaciones colectivas entre empresas. Seminario Iboamericano sobre Integración Empresarial y Cooperativa: cooperar $y$ exportar para ganar.

10. ISOTools. (2017). Mejora de los procesos internos. http://cort.as/-SIMd

11. Lahaba, Y. N., \& Santos, M. L. (2001). La gestión del conocimiento: una nueva perspectiva. ACIMED. http://cort.as/-Sjjt

12. Moncayo, C. (14 de septiembre de 2017). Instituto Nacional de Contadores
Públicos. Colombia. https://www.incp. org.co/las-pymes-la-verdadera-locomotora-economica-colombiana/

13. Nonaka, I., \& Takeuchi, H. (1995). The knowledge-creating company: How japanese companies create the dynamics of innovation. New York.

14. Organización de las Naciones Unidas (ONU). (2019). Día de las Microempresas y las Pequeñas y Medianas Empresas. https:// www.un.org/es/events/smallbusinessday/

15. Organización Internacional del Trabajo (OIT). (2019). Global evidence on the contribution to employment by the self-employed, micro-enterprises and SMEs. http://cort.as/-St7j

16. Quinn, R. (1983). A spatial model of effectiveness criteria: Towards a competing values approach to organizational analysis. Management Science, 29(3), 363-377.

17. Revista Dinero. (2018). Los retos que enfrentan las mipymes en Colombia. https://www.semana.com/edicion-impresa/ pymes/articulo/los-retos-que-enfrentan-las-mipymes-en-colombia/241586/

18. Sabogal, J. (30 de octubre de 2018). RCN Radio. http://cort.as/-ISo5

19. Sepúlveda, C. (1995). Diccionario de Términos Económicos. http://cort.as/-SIK5

20. Unipymes. (26 de octubre de 2018). Las pymes deben mejorar el uso de la productividad de sus activos: IARA. http://cort.as/-Sv3f

21. Velásquez, F. (2017). La estrategia, la estructura y las formas de asociación: fuentes de ventaja competitiva para las $P y$ mes colombianas. Estudios Gerenciales.

\section{Apéndices o anexos}

Anexo A. Cuestionario

https://drive.google.com/file/d/ 1 h CRSzhkMFYCOWfRF w W Xi Q_ 9 N M 2 m_ G n K G / 